\title{
Evaluation of diaphragm in chronic obstructive pulmonary disease patients using ultrasonography in relation to disease severity.
}

Sherif R. Abdel Fattah ${ }^{(1)}$, Radwa A. Elhefny ${ }^{(2)}$, Enas S. Farhat ${ }^{(3)}$, Yousra S. Fathy ${ }^{(4)}$

1) Professor of chest diseases and tuberculosis, Faculty of Medicine, Fayoum University

2)ass. Professor of chest diseases and tuberculosis, Faculty of Medicine, Fayoum University

3) Lecturer of chest diseases and tuberculosis, Faculty of Medicine, Fayoum University

4) Demonstrator of chest diseases and tuberculosis, Faculty of Medicine, Fayoum University

Corresponding Author: Yousra sayed Fathy

Email Address: ysf00@ fayoum.edu.eg

Tel: 01097809106

\begin{abstract}
Back Ground: The diaphragm is considered a major respiratory muscle in which any alterations in either structure or function in stable COPD patients may cause a significant adverse clinical consequence. (1) Evaluation of the diaphragm using ultrasonography is considered a simple, non-invasive, available bedside technique that has been used increasingly both in clinical and research settings. (2)

Aim of Work: ultrasonographic evaluation of the diaphragm in COPD patients and its relation to disease severity.
\end{abstract}

\section{Patients and methods:}

The study was carried out during the period from December 2019-

December 2020 on forty patients with clinically stable COPD during their follow up in the outpatient clinic of the chest department, Fayoum University hospital.

\section{Results:}

The diaphragmatic excursion assessed by ultrasonography was found to decrease progressively with increasing COPD severity.

\section{Conclusion:}

Ultrasonography is a reliable, noninvasive, and simple method that can be used for the evaluation of diaphragmatic excursion and a negative correlation was found between diaphragmatic excursion assessed by ultrasonography and COPD severity.

Keywords ultrasonography, diaphragmatic Excursion, chronic obstructive pulmonary disease severity. 


\section{Introduction:}

Chronic obstructive pulmonary disease is a widespread disease that can be both prevented and treated, it's characterized by permanent respiratory symptoms and airflow limitation caused by airway and /or alveolar disorders typically due to significant exposure to harmful gases or particles, and it's also affected by factors related to the host such as lung development abnormality [3].

COPD is considered a systemic inflammatory disease so it can cause impacts on several systems such as skeletal muscle dysfunction and weight loss [4].

In addition to affecting the peripheral musculature, muscle fatigue caused by the harmful effect of COPD may impair respiratory muscle function [5].

The diaphragm is the major respiratory muscle used for quiet breathing. Different structural and functional techniques are available for evaluating the diaphragm [6]. Recently diaphragmatic ultrasound evaluation has gained importance as a safe, radiation-free, bedside tool to study diaphragm function [7]. Ultrasound can provide both morphological and functional information, then allowing to repeat measurement over time by both B.mode, M.mode [8].

\section{Patients and methods:}

A cross sectional study was conducted in the chest department, Fayoum University hospital from December 2019 to December 2020 for ultrasonographic assessment of diaphragmatic excursion and its relation COPD severity.

\section{Patients' selection:}

The study included forty patients with COPD who were diagnosed and classified into four groups according to GOLD 2020 as following: The first group: included 10 patients with a mild stage. The second group: included 10 patients with a moderate stage. The third group: included 10 patients with a severe stage. The fourth group: included 10 patients with a very severe stage. The study also included forty healthy individuals of the same age and sex as COPD patients.

\section{Inclusion criteria:}

1-stable COPD patients aged more than 40 years who were diagnosed and classified into four groups of severity according to GOLD 2020.

2- controls will be healthy individuals who never smoked.

\section{Exclusion criteria:}

Subjects who had any of the following conditions were excluded from the study:

1-patient with a known neuromuscular disorder.

2- patient with known anatomical malformation of the diaphragm.

3 -post abdominal or thoracic surgery 


\section{Methods:}

All participants gave their informed consent for inclusion before sharing in the study. Every person was submitted to:

\section{1. full medical history}

2. clinical examination

3. chest $x$-ray

4. Spirometry
5. 6MWT (six-minute walk test)

6. chest ultrasonography: An ultrasound machine (Philips Clear Vue 350) was used to assess the diaphragmatic excursion at TLC through the low-frequency curvilinear probe (4 MHz).

\section{Techniques:}

Patients were examined in the semisitting position. The examination was performed to the right hemidiaphragm through the liver window due to difficult visualization of the left hemidiaphragm caused by the presence of the smaller splenic window.

\section{Assessment of diaphragmatic excursion (DE):}

- The lower frequency curvilinear probe was placed subcostally parallel to the intercostal space between the midclavicular and anterior axillary lines, "anterior subcostal view".

- In B-mode ultrasonography the diaphragm was seen as a hyperechogenic line surrounding the liver. At this point, the probe was directed medially, cranially, and dorsally to visualize the posterior third of the right diaphragm.

- Then Imaging was changed to M-mode with the line of sight positioned to obtain maximum excursion.

- in M-mode, the diaphragm was visualized as a hyperechogenic line that assumed in time a sinusoidal form with the peak corresponding to maximum inspiration and the trough corresponding to expiration.

- the diaphragmatic excursion on this M-mode trace was measured as it represented the height of the curve [9].

- Ethical consideration:

- This study was reviewed by the Faculty of Medicine Research Ethical Committee. The participants were informed about the objectives of the study, the examination, the 
investigation that will be done, and the confidentiality of their information, and their right not to participate in the study.

\section{- Statistical methods:}

- Data were coded and entered using the statistical package for the Social Sciences (SPSS) version 26 (IBM Corp., Armonk, NY, USA). Data were summarized using mean and standard deviation for quantitative variables and frequencies (number of cases) and relative frequencies (percentages) for categorical variables. Comparisons between groups were done using unpaired t-test when comparing 2 groups and analysis of variance (ANOVA) with multiple comparisons post hoc test when comparing more than 2 groups [10]. For comparing categorical data, Chi-square $(\chi 2)$ test was performed. An exact test was used instead when the expected frequency is less than 5 [11]. Correlations between quantitative variables were done using the Pearson correlation coefficient [12]. P-values less than 0.05 were considered as statistically significant.

\section{Results:}

In this study, 80 male patients were included in the form of 40 COPD patients diagnosed and classified according to GOLD 2020 as follows: 10(25\%) as mild, $10(25 \%)$ as moderate, $10(25 \%)$ as severe, $10(25 \%)$ as very severe and another 40 individuals served as the control group with no significant statistical difference between the patients and the control group $(\mathbf{P}<\mathbf{0 . 0 0 9})$ with a mean age of the cases $(59.18 \pm 4.93)$, while that of the control group was $(56.38 \pm 4.48)$. 
Table (1): statistical comparison between cases and control excursion.

\begin{tabular}{|l|c|c|c|c|c|c|c|c|c|c|}
\hline & \multicolumn{4}{|c}{ cases } & \multicolumn{3}{|c|}{ control } & P-value \\
\hline $\begin{array}{l}\text { Diaphragmatic } \\
\text { Excursion (cm) }\end{array}$ & 3.91 & 0.62 & 2.50 & 5.05 & 6.30 & 0.42 & 5.25 & 6.90 & $<0.001$ \\
\hline
\end{tabular}

The table illustrates a statistical comparison between the study group and control showing that there was a highly significant statistical difference between cases and control as regard diaphragmatic excursion) $(\mathbf{P}<\mathbf{0 . 0 0 1})$ with lower values in the cases, with mean value in the COPD patients being (3.91 \pm 0.62$)$ While the mean value in the control group of diaphragmatic excursion was (6.3 \pm 0.42$)$.

Table (2): statistical comparison among COPD patients with different grades of severity as regard Diaphragmatic excursion.

\begin{tabular}{|c|c|c|c|c|c|c|c|c|c|}
\hline & \multicolumn{8}{|c|}{ GOLD severity } & \multirow{3}{*}{$\begin{array}{c}\text { P- } \\
\text { valu } \\
\text { e }\end{array}$} \\
\hline & \multicolumn{2}{|c|}{ mild } & \multicolumn{2}{|c|}{ moderate } & \multicolumn{2}{|c|}{ severe } & \multicolumn{2}{|c|}{ very severe } & \\
\hline & $\begin{array}{c}\text { Mea } \\
\text { n }\end{array}$ & SD & Mean & SD & Mean & SD & Mean & SD & \\
\hline $\begin{array}{l}\text { Diaphragmati } \\
\text { c Excursion } \\
(\mathrm{cm})\end{array}$ & 4.59 & 0.24 & 4.20 & 0.23 & 3.75 & 0.26 & 3.09 & 0.29 & $\begin{array}{c}< \\
0.00 \\
1\end{array}$ \\
\hline
\end{tabular}

Table showing highly significant statistical difference $(\mathbf{P}<\mathbf{0 . 0 0 1})$ between COPD patients with different grades of severity as regard diaphragmatic excursion with the lowest values in the very severe group. 


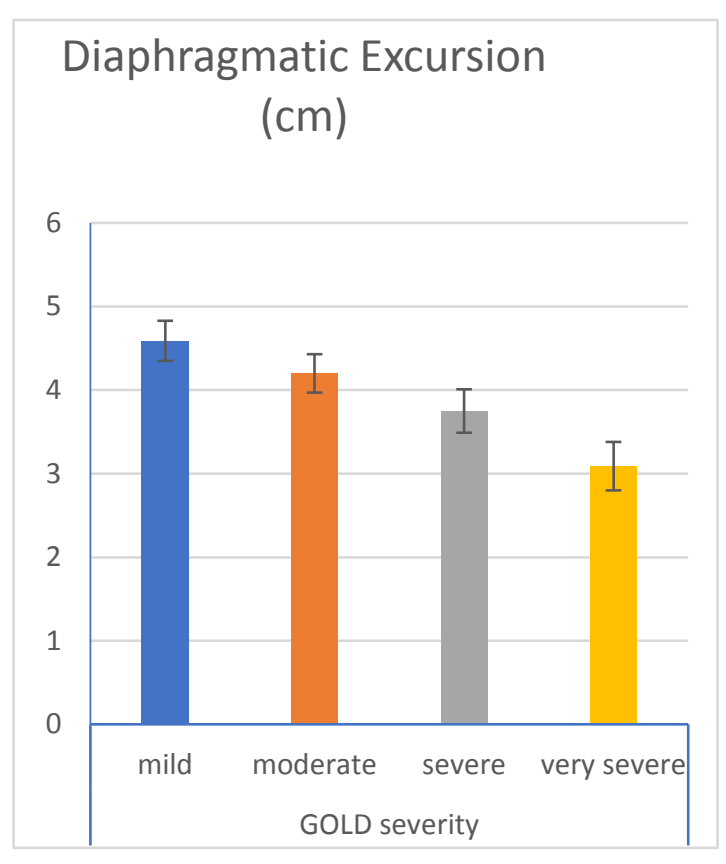

Figure (1): statistical comparison between COPD patients with different grades of severity regarding diaphragmatic excursion.

\section{Discussion:}

Ultrasonography provides a simple, safe, and non-invasive method for assessment and visualization of diaphragmatic function and mobility. The primary goal of our study was to determine the diaphragmatic mobility in patients with COPD compared with normal controls and comparing them between different grades of severity in COPD patients.[13]. in the present study, 80 patients have been included 40 COPD patients and 40 controls all of them were males. with a mean age of the cases $(59.18$ \pm 4.93 ), while that of the control group was $(56.38 \pm 4.48)$.

In our study, there was a highly significant difference $(\mathrm{p}<0.001)$ between the cases and control as regards diaphragmatic excursion, With lower values in the COPD patients.

This agreed with the study by Abd El-hay Ibrahim Abd El-hay, et al;2019 [13] who found that the control showed a higher diaphragmatic excursion than the COPD group. 
It also agreed with Camilo

Corbellini, et al;2018[14] who found that the diaphragmatic mobility showed significant differences between the COPD patients and the healthy controls and that Diaphragmatic mobility during deep inspiration lower than in the healthy controls.

Davachi, et al;2014[15], Paulin, et al;2007[16], Dos Santos Yamaguti, et al;2008[17]. agreed with our study as they reported statistically significant differences regarding diaphragmatic motility measured by the US, between the patients and control. In our study, there was a highly significant statistical difference in diaphragmatic excursion between different grades of COPD severity with decreasing values with the disease progression

This study agreed with Ayman Amin and Moustafa Zedan,2018[18] and Dos Santos Yamaguti, et al; 2008 [17]who reported that Diaphragmatic excursion during deep breathing correlated significantly with disease severity.

\section{References:}

1. Bharat Bhushan Sharma and Virendra Singh. Diaphragmatic dysfunction in chronic obstructive pulmonary disease, Lung India. 2019; Jul-Aug; 36(4): 285287.

2. Zambon $\mathrm{M}$, Greco $\mathrm{M}$, Bocchino $\mathrm{S}$, Zangrillo A, et al. Assessment of

diaphragmatic dysfunction in the critically ill patient with ultrasound: A systematic review. Intensive Care Med. 2017; 43: 29-38.

3. Global Initiative for chronic obstructive lung disease 2020. 
4. Barreiro, E and Gea, J.Respiratory and limb muscle dysfunction in COPD. COPD: Journal of Chronic Obstructive Pulmonary Disease. 2015;12(4),413426.

5. Barreiro, E and Jaitovich, A. Muscle atrophy in chronic obstructive pulmonary disease: Molecular basis and potential therapeutic targets. Journal of Thoracic Disease. 2018;10(Suppl 12), S1415-S1424.

6. Gerscovich EO, Cronan M, McGahan JP, Jain K, Jones CD and McDonald C.ultrasonographic evaluation of diaphragmatic motion. J Ultrasound Med. 2001; 20:597-604.

V.Matamis D, Soilemezi E, Tsagourias M, Akoumianaki E, Dimassi S, Boroli F, et al.Sonographic evaluation of the diaphragm in critically ill patients. Technique and clinical applications. Intensive Care Med. 2013; 39(5):801-10.

8.Ferrari G, De Filippi G, Elia F, Panero F and Volpicelli G. Diaphragm ultrasound as a new index of discontinuation from mechanical ventilation. Crit Ultrasound J. 2014; 6:8.

9.Testa A, Soldati $G$ and Giannuzi R. (Ultrasound M-mode assessment of diaphragmatic kinetics by anterior transverse scanning in healthy subjects. Ultrasound Med Biol. 2011;37: 44 -52.

10.Chan YH (2003a): Biostatistics 102: Quantitative Data - Parametric \& Nonparametric Tests. Singapore Med J.;44(8): 391-396.

11.Chan YH (2003b): Biostatistics 103: Qualitative Data -Tests of Independence. Singapore Med J.;44(10): 498-503.

12.Chan YH (2003c): Biostatistics 104:

Correlational Analysis. Singapore Med J.;44(12): 614-619.

13.Abd El-hay Ibrahim Abd El-hay, Houssam Eldin Hassanin Abd Elnaby and Mohamed Mohy Mohamed Erfan El-Gama. Ultrasonographic Assessment of Diaphragmatic Function and Its Correlation with Chronic Obstructive Pulmonary Disease Severity, The Egyptian Journal of Hospital Medicine (July) 2019; Vol. 76 (7), Page 4524-4532 4524.

14.Camilo Corbellini, Alain Boussuges, Jorge Hugo Villafañe and Luciano Zocchi.Diaphragmatic Mobility Loss in Subjects with Moderate to Very Severe COPD May Improve After In-Patient Pulmonary Rehabilitation. Respiratory Care. 2018; October 63 (10) 1271-1280. 
15.Davachi B, Lari SM, Attaran D, et al.

The relationship between diaphragmatic

movements in sonographic assessment and

disease severity in patients with stable

Chronic Obstructive Pulmonary Disease

(COPD). J Thorac. 2014; 2(3):187-192.

16. Paulin E, Yamaguti WP, Chammas MC,

Shibao S, Stelmach R, Cukier A et al.

Influence of diaphragmatic mobility on

exercise tolerance and dyspnea in patients

with COPD. Respir Med. 2007; 101:2113-

2118.

17.Dos Santos Yamaguti, W.P, Paulin, E,

Shibao, S, et al. Air trapping: The major

factor limiting diaphragm mobility in

chronic obstructive pulmonary disease

patients. Respirology. 2008;13, 138-144.

18. Ayman Amin and Moustafa Zedan.

Transthoracic ultrasonographic evaluation of

diaphragmatic excursion in patients with

chronic obstructive pulmonary disease.

Ejbronchology. 2018;12: 27-32. 